Collingwood, E. F., and G. Piranian

Math. Zeitschr. 84, 246-253 (1964)

\title{
Tsuji functions with segments of Julia *
}

\author{
Dedicated to Helmut Grunsky on his $60^{\text {th }}$ Birthday, $11^{\text {th }}$ July 1964 \\ By \\ E. F. Collingwood and George Piranian
}

\section{Introduction}

Let $D$ denote the unit disk $|z|<1, C$ the unit circle $|z|=1$, and $C_{r}$ the circle $|z|=r$. Corresponding to any function $w$ meromorphic in $D$ we denote by $w^{*}$ the spherical derivative:

$$
w^{*}(z)=\frac{\left|w^{\prime}(z)\right|}{1+|w(z)|^{2}} .
$$

We say that $w$ is a Tsuji function provided the spherical length of the curve $w\left(C_{r}\right)$ is a bounded function in $0<r<1$, in other words, provided

$$
\sup _{r<1} \int_{0}^{2 \pi} w^{*}\left(r e^{i \vartheta}\right) r d \vartheta<\infty .
$$

A rectilinear segment $S$ lying in $D$ except for one endpoint $e^{i \vartheta}$ on $C$ is called a segment of Julia for $w$, provided in each open triangle in $D$ having one vertex at $e^{i \vartheta}$ and meeting $S$, the function $w$ assumes all values on the Riemann sphere except possibly two. A point $e^{i \vartheta}$ is a Julia point for $w$ provided each rectilinear segment lying in $D$ except for one endpoint at $e^{i \vartheta}$ is a segment of Julia for $w$.

Corresponding to each $\vartheta$ and each $\alpha(|\alpha|<\pi / 2)$, let $S(\vartheta, \alpha)$ be the segment that joins the points $e^{i \vartheta}$ and $\left(1-e^{i \alpha} \cos \alpha\right) e^{i \vartheta}$; in other words, let $S(\vartheta, \alpha)$ denote the chord of the circle with diameter $\left[0, e^{i \theta}\right]$ that forms a directed angle $\alpha$ with $\left[0, e^{i \vartheta}\right]$ at $e^{i \vartheta}$. In case $w(z)$ approaches a limit as $z \rightarrow e^{i \vartheta}$ on $S(\vartheta, \alpha)$, we denote this limit by $w(\vartheta, \alpha)$.

The present note answers a question that W. SeIDel raised concerning a theorem of M. TsuJI [3]. In terms of the notation introduced in the preceding paragraph, we can state Tsuji's theorem as follows.

Let $w$ be a Tsuji function, and let $\Lambda(\vartheta, \alpha)$ denote the spherical length of the image under $w$ of $S(\vartheta, \alpha)$. Then, for each $\alpha$ in $|\alpha|<\pi / 2, \Lambda(\vartheta, \alpha)$ is an integrable function of $\vartheta$; and for almost all $\vartheta, \Lambda(\vartheta, \alpha)$ is an integrable function of $\alpha$. Moreover, for all $\vartheta$ in a set of measure $2 \pi$ on $[0,2 \pi)$, the relation $w(\vartheta, \alpha)=w(\vartheta, \beta)$ holds whenever both limits exist, while $S(\vartheta, \gamma)$ is a segment of Julia if $\Lambda(\vartheta, \gamma)=\infty$. In particular, the theorem implies that if $w$ is a Tsuji function, then $\Lambda(\vartheta, \alpha)<\infty$ except for a set of points $(\vartheta, \alpha)$ of two-dimensional measure 0 .

* This paper was written with partial support from the National Science Foundation (Grant G-23830). 
In a review of [3], SEIDEL asked whether the segments of Julia mentioned in the theorem can actually occur. We shall display several relevant examples.

\section{Meromorphic Tsuji functions}

Lemma. If $\left\{z_{n}\right\}$ is a sequence of points in the unit disk $D$ such that $\left|z_{n+1}\right|>\left|z_{n}\right|$ for all $n$ and $z_{n} \rightarrow 1$ as $n \rightarrow \infty$, then the function

$$
w(z)=\sum a_{n} /\left(z-z_{n}\right)
$$

is a Tsuji function provided the $a_{n}$ are small enough.

Proof. We chose a sequence $\left\{\rho_{n}\right\}$ of positive numbers such that $\rho_{n+1}+$ $\rho_{n}<\left|z_{n+1}\right|-\left|z_{n}\right|$ for all $n$, and we denote by $D_{n}$ the disk $\left|z-z_{n}\right|<\rho_{n}$. Clearly, $\sum \rho_{n}<1$.

Now let $\left\{a_{n}\right\}$ denote any sequence subject to the restriction that $0<a_{n}<\rho_{n}^{3}$ for all $n$. Then

$$
\sum^{*} \frac{a_{n}}{\left|z-z_{n}\right|}<\sum \frac{a_{n}}{\rho_{n}}<\sum \rho_{n}^{2}<1
$$

and

$$
\sum^{*} \frac{a_{n}}{\left|z-z_{n}\right|^{2}}<\sum_{n} \frac{a_{n}}{\rho^{2}}<\sum \rho_{n}<1,
$$

where the asterisk indicates that if $z$ lies in $D_{m}$, the $m$-th term is to be omitted.

If a circle $C_{r}$ meets none of the disks $D_{n}$, it follows from (3) that $\left|w^{\prime}(z)\right|<1$ on $C_{r}$, and hence that $w\left(C_{r}\right)$ has Euclidean length less than $2 \pi$.

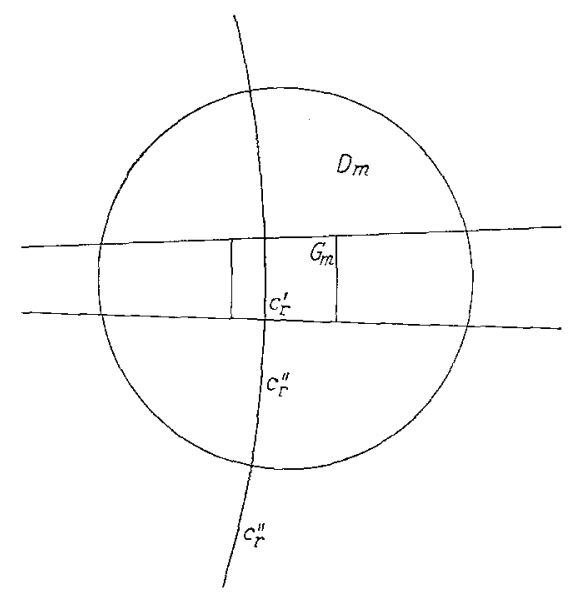

Fig. 1

If $C_{r}$ meets the set $\cup D_{n}$, it meets precisely one of the disks, say $D_{m}$. For the sake of typographical convenience we suppose that $z_{m}$ is real and that $\frac{1}{2}<z_{m}<1$ (so that $a_{m}<\frac{1}{8}$ ). We denote by $G_{m}$ the rectangle

$$
\left\{z\left|z=r e^{i \vartheta},\right| r-z_{m}\left|\leqq a_{m} / 3,\right| \vartheta \mid \leqq a_{m} / 3\right\} ;
$$

we write $C_{r}^{\prime}=C_{r} \cap G_{m}$ and $C_{r}^{\prime \prime}=C_{r} \backslash C_{r}^{\prime}$ (Fig. 1 shows the relation - not to scale - between $D_{m}, G_{m}$, and $C_{r}$; note that $C_{r}^{\prime}$ is empty if $z_{m}-\rho_{m}<r<z_{m}-a_{m} / 3$ or $\left.z_{m}+a_{m} / 3<r<z_{m}+\rho_{m}\right)$; and we estimate separately the spherical length of $w\left(C_{r}^{\prime}\right)$ and the Euclidean length of $w\left(C_{r}^{\prime \prime}\right)$.

On $C_{r}^{\prime}$, we use the relations

$$
\left|z-z_{m}\right|^{2}=r^{2}+z_{m}^{2}-2 r z_{m} \cos \vartheta=\left(r-z_{m}\right)^{2}+4 r z_{m} \sin ^{2} \vartheta / 2<2 a_{m}^{2} / 9 .
$$

They imply that $a_{m} /\left|z-z_{m}\right|>2$; together with (2), this gives the inequalities

$$
|w(z)|>\frac{a_{m}}{\left|z-z_{m}\right|}-\sum^{*} \frac{a_{n}}{\left|z-z_{n}\right|}>\frac{a_{m}}{\left|z-z_{m}\right|}-\sum \frac{a_{n}}{\rho_{n}}>\frac{a_{m}}{2\left|z-z_{m}\right|} .
$$


Also, for points $z$ on $C_{r}^{\prime} \backslash\left\{z_{m}\right\}$ it follows from (3) that

$$
\left|w^{\prime}(z)\right|<\frac{a_{m}}{\left|z-z_{m}\right|^{2}}+\sum^{*} \frac{a_{n}}{\left|z-z_{n}\right|^{2}}<\frac{a_{m}}{\left|z-z_{m}\right|^{2}}+1<\frac{2 a_{m}}{\left|z-z_{m}\right|^{2}},
$$

and we deduce that

$$
w^{*}(z)<\left|w^{\prime}(z)\right| \cdot|w(z)|^{-2}<8 / a_{m}
$$

on $C_{r}^{\prime}$. Since $C_{r}^{\prime}$ has length less than $2 a_{m} / 3$, the curve $w\left(C_{r}^{\prime}\right)$ has spherical length less than $\frac{16}{3}$.

The arc $C_{r}^{\prime \prime}$ may contain a subarc of points $z=r e^{i \vartheta}$ with $-a_{m} / 3<\vartheta<a_{m} / 3$. If that is the case, the inequalities

$$
\left|w^{\prime}(z)\right|<1+a_{m} /\left|z-z_{m}\right|^{2}<1+1 / 9 a_{m}
$$

hold on the subarc, and therefore $w$ maps the subarc onto an arc of Euclidean length less than

$$
\frac{2}{3} a_{m}\left(1+\frac{1}{9 a_{m}}\right)=\frac{2}{3} a_{m}+\frac{2}{27}<\frac{1}{6} .
$$

For the remainder of $C_{r}^{\prime \prime}$, we use the relations

$$
\left|r e^{i \vartheta}-z_{m}\right|^{2}=\left(r-z_{m}\right)^{2}+4 r z_{m} \sin ^{2} \vartheta / 2 \geqq 4 r z_{m} \vartheta^{2} / \pi^{2}>2 r \vartheta^{2} / \pi^{2} .
$$

They yield the upper bound

$$
\begin{aligned}
2 \int_{a_{m} / 3}^{\pi}\left|w^{\prime}\left(r e^{i \vartheta}\right)\right| r d \vartheta & <2 \int_{a_{m} / 3}^{\pi}\left(1+\frac{a_{m}}{\left|r e^{i \vartheta}-z_{m}\right|^{2}}\right) r d \vartheta \\
& <2 \pi+a_{m} \pi^{2} \int_{a_{m} / 3}^{\pi} \vartheta^{-2} d \vartheta<2 \pi+3 \pi^{2} .
\end{aligned}
$$

In summary: the spherical length of $w\left(C_{r}\right)$ is less than $2 \pi+3 \pi^{2}+\frac{17}{3}$, and the lemma is established.

Theorem 1. There exists a Tsuji function for which each point $e^{i g}$ is a Julia point.

Proof. Let $z_{n}=\left(1-n^{-\frac{1}{2}}\right) e^{i \log n}(n=2,3, \ldots)$, and choose the constants $a_{n}$ as in the proof of the lemma. Then the function (1) is a Tsuji function.

Since the right member of (1) converges uniformly in the complement $H$ (relative to the plane) of the set $\cup D_{n}$, it defines a function $w$ that is continuous on $H$. Now let $S$ denote a line segment in $D$, with an endpoint $e^{i \vartheta}$, and let $A$ denote a Stolz angle containing $S$. Then there exist infinitely many integers $n_{k}$ such that the disk $D_{n_{k}}$ lies in $A$. For large $k$, the set of values omitted by $w$ in $D_{n_{k}}$ lies in a small neighborhood of the point $w\left(e^{i \vartheta}\right)$, and therefore $S$ is a segment of Julia. This completes the proof of Theorem 1.

The following theorem shows that segments of Julia may occur even if all segmental limits $w(\vartheta, \alpha)$ exist. 
Theorem 2. There exists a Tsuji function $w$ with the following two properties:

(i) If $S$ is a chord of the unit disk, then the spherical length of the arc $w(S)$ is less than some constant independent of $S$.

(ii) The radius of the point 1 is a segment of Julia for $w$.

Proof. First we choose the points $z_{n}$ so that they lie on the parabola $y=$ $(x-1)^{2}$; then we select the constants $\rho_{n}$ small enough so that no line meets more than two of the disks $D_{n}$. The remainder of the proof follows the pattern that we have already established.

Theorem 3. If $E$ is a set of measure 0 on $C$, then there exists a Tsuji function of bounded characteristic for which every point of $E$ is a Julia point.

Proof. Since $E$ has measure 0 , we can choose a sequence of $\operatorname{arcs} A_{m}$ on $C$, of lengths $\sigma_{m}$ and with midpoints $t_{m}$, such that each point of $E$ lies in infinitely many of the arcs $A_{m}$ and such that $\sum \sigma_{m}<\infty$. For each $m$, we denote by $J_{m}$ the intersection of $D$ with the circle $\left|z-t_{m}\right|=\sigma_{m}$.

There exists a sequence $\left\{k_{m}\right\}$ of positive integers such that $k_{m} \rightarrow \infty$ and $\sum k_{m} \sigma_{m}<\infty$. If on each arc $J_{m}$ we choose $k_{m}$ equally spaced points $\zeta_{m n}$ (in such a way that the angular distance between $\zeta_{m n}$ and $\zeta_{m, n+1}$ is approximately $\pi / k_{m}$ ), then, at each point of $E$, every Stolz angle contains infinitely many of the points $\zeta_{m n}$. We can easily choose the $\zeta_{m n}$ in such a way that $\left|\zeta_{m_{1} n_{1}}\right|+\left|\zeta_{m_{2} n_{2}}\right|$ except when $m_{1}=m_{2}$ and $n_{1}=n_{2}$, and therefore we can choose disks $D_{m n}$ with centers $\zeta_{m n}$ in such a way that no circle $C_{r}$ meets more than one of the disks. We now form two Blaschke products $B_{1}(z)$ and $B_{2}(z)$, the first with zeros $b_{m n}=$ $\left(1+\varepsilon_{m n}\right) \zeta_{m n}$, the second with zeros $c_{m n}=\left(1-\varepsilon_{m n}\right) \zeta_{m n}$. If the $\varepsilon_{m n}$ are sufficiently small, then each pair of zeros lies close to the center of the corresponding disk. The convergence of the two products follows from the inequality

$$
\sum_{m, n}\left(1-\left|\zeta_{m n}\right|\right)<\sum_{m=1}^{\infty} k_{m} \sigma_{m} .
$$

Now let $w(z)=B_{1}(z) / B_{2}(z)$. If the $\varepsilon_{m n}$ are sufficiently small, the product

$$
\prod_{m, n} \frac{b_{m n}-z}{1-\bar{b}_{m n} z} \cdot \frac{1-\bar{c}_{m n} z}{c_{m n}-z}
$$

converges uniformly in $\bar{D} \backslash \cup D_{m n}$, and therefore the symbol $w\left(e^{i \vartheta}\right)$ has a meaning. Moreover, if $\varepsilon_{m n} \rightarrow 0$ fast enough as $m \rightarrow \infty$, then for any sequence of disks $D_{m n}$ tending to a point $e^{i \vartheta}$, the set of values omitted by $w$ in $D_{m n}$ lies in a small neighborhood of $w\left(e^{i \vartheta}\right)$ when $m$ is large. Therefore every segment in $D$ terminating at a point of $E$ is a segment of Julia for $w$.

To see that $w$ is a Tsuji function, we note that if the $\varepsilon_{m n}$ are small enough, then $w^{\prime}(z)$ is bounded in the set $D \backslash \cup D_{m n}$, and that in $D_{m n}$ the function $w$ is the product of the factor with index $(m, n)$ in (4) and a function whose values lie in an annulus $R_{1}<|z|<R_{2}\left(R_{1}\right.$ and $R_{2}$ positive, independent of $m$ and $\left.n\right)$ and whose derivative is bounded. 
We observe that since the function is of bounded characteristic, the set of its Fatou points (at each of which it has a uniform limit in every Stolz angle) is of measure $2 \pi$ on $C$, so that the set of its Plessner points on $C$ (at each of which the cluster set of the function in every Stolz angle is total) is of zero measure, by PLESSNER's theorem [2, p. 70]. Since the Julia points form a subset of the Plessner points, the property of the set $E$ in Theorem 3 is best possible.

\section{The Tsuji set of a meromorphic function}

Let $w$ denote any meromorphic function in the unit disk $D$; corresponding to each point $\alpha$ in $D$, we write

$$
w_{\alpha}(z)=w\left(\frac{z-\alpha}{1-\bar{\alpha} z}\right),
$$

and we define the Tsuji set of $w$ to be the set of values $\alpha$ for which $w_{\alpha}$ is a Tsuji function. Since the quantity

$$
\sup _{r<1}^{2 \pi} \int_{0}^{*} w_{\alpha}^{*}\left(r e^{i \vartheta}\right) r d \vartheta
$$

is a lower-semicontinuous function of $\alpha$, the Tsuji set of a meromorphic function is a point set of type $F_{\sigma}$.

Theorem 4. The Tsuji set of a function may be the point set $D \backslash\{0\}$.

Proof. For $k=2,3, \ldots$, we choose $k$ equally spaced points $z_{n k}$ on the circle $|z|=1-b_{k}$, where $\left\{b_{k}\right\}$ is a strictly decreasing sequence with $b_{1}<1, b_{k} \rightarrow 0$. We construct the function (1) as in the proof of the lemma, except that now some circles $C_{r}$ meet several of the disks $D_{n k}$. If the $b_{k}$ and the $a_{n k}$ are small enough, then the spherical length of the curve $w\left(C_{r}\right)$, where $r=1-b_{k}$, has the order of magnitude $\pi k$, and therefore $w$ is not a Tsuji function. On the other hand, if $b_{k+1} \mid b_{k} \rightarrow 0$ fast enough, then for each $\alpha$ in $D \backslash\{0\}$ the number of disks that meet the circle $|(z-\alpha) /(1-\bar{\alpha} z)|=r$ is a bounded function of $r$ (in fact, it is 1 or 0 , for $r>r_{z}$ ), and therefore $w_{\alpha}$ is a Tsuji function for all $\alpha$ except $\alpha=0$.

\section{Holomorphic Tsuji functions}

Theorem 5. There exist holomorphic Tsuji functions with segments of Julia.

If the function $w$ constructed in the proof of Theorem 2 were to omit the value $w(1)$, in $D$, then the function $1 /[w(z)-w(1)]$ would provide a proof of Theorem 5. However, it is not obvious that the $a_{n}$ can always be chosen so that $w$ omits the value $w(1)$. In particular, if the $z_{n}$ and the $a_{n}$ are real, then $w(1)$ is real, and $w$ assumes this value in each interval $\left(z_{n}, z_{n+1}\right)$.

We shall prove that the function

$$
w(z)=\exp \left(\frac{1+z}{1-z}\right)^{2}
$$


is a Tsuji function with two segments of Julia. First we observe that if $r$ is near to 1 , the mapping

$$
f(z)=\frac{1+z}{1-z}
$$

carries the circle $C_{r}$ onto a large circle a long arc of which lies near the imaginary axis; that the function $\exp f(z)$ carries this arc onto an arc making many turns around the unit circle $C$, not far from $C$; and that $\exp f(z)$ is therefore not a Tsuji function. On the other hand, the mapping $g(z)=[f(z)]^{2}$ carries $C_{r}$ onto a reniform curve $\Gamma_{r}$ that meets the imaginary axis in four points, each time at an angle of approximately $\pi / 4$ (Fig. 2 shows approximately the upper half of the circle $f\left(C_{r}\right)$ and the curve $\Gamma_{r}$, for $\left.r=\frac{1}{2}\right)$. The function $w(z)=\exp g(z)$ therefore carries $C_{r}$ onto a curve

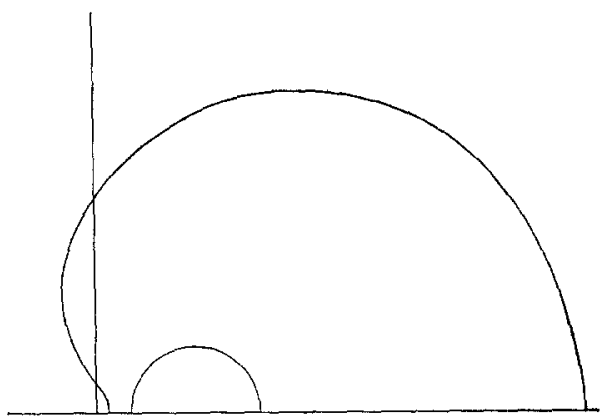

Fig. 2 making few turns around the origin, except quite near the origin and quite far from the origin; that is, the majority of the turns of the image of $C_{r}$ make only small contributions to the spherical length of the image. We shall now show that $w$ is indeed a Tsuji function.

Since

$$
\left|w^{\prime}(z)\right|=\left|\frac{4(1+z) w}{(1-z)^{3}}\right| \text { and } w^{*}(z)<\left|\frac{4(1+z)}{(1-z)^{3} w}\right|
$$

it will be convenient to integrate the first or the second expression, on subarcs of $C_{r}$, according as the real part of $g(z)$ is negative or positive on these subarcs. Now, at $z=r e^{i \theta}$,

and

$$
\left|\frac{1+z}{(1-z)^{3}}\right|=\frac{\left[1+r^{2}+2 r \cos \vartheta\right]^{\frac{1}{2}}}{\left[1+r^{2}-2 r \cos \vartheta\right]^{\frac{1}{2}}}=\frac{\left[(1+r)^{2}-4 r \sin ^{2} \vartheta / 2\right]^{\frac{1}{3}}}{\left[(1-r)^{2}+4 r \sin ^{2} \vartheta / 2\right]^{\frac{3}{3}}}
$$

$$
\begin{aligned}
|w(z)| & =\exp \Re\left(\frac{1+z}{1-z}\right)^{2}=\exp \Re\left(\frac{1-r^{2}+2 i r \sin \vartheta}{(1-r)^{2}+4 r \sin ^{2} \vartheta / 2}\right)^{2} \\
& =\exp \frac{\left(1-r^{2}\right)^{2}-4 r^{2} \sin ^{2} \vartheta}{\left[(1-r)^{2}+4 r \sin ^{2} \vartheta / 2\right]^{2}} .
\end{aligned}
$$

In view of the symmetry of $w\left(C_{r}\right)$, it will be sufficient to show that the integral

$$
\int_{0}^{\pi} \frac{4\left[(1+r)^{2}-4 r \sin ^{2} \vartheta / 2\right]^{\frac{2}{2}}}{\left[(1-r)^{2}+4 r \sin ^{2} \vartheta / 2\right]^{\frac{3}{2}}} \exp \left\{-\frac{\left|\left(1-r^{2}\right)^{2}-4 r^{2} \sin ^{2} \vartheta\right|}{\left[(1-r)^{2}+4 r \sin ^{2} \vartheta / 2\right]^{2}}\right\} d \vartheta
$$


is a bounded function of $r$, for $\frac{1}{2}<r<1$. Over the interval $\pi / 4 \leqq \vartheta \leqq \pi$, the integrand has a bound independent of $r$, and therefore we may restrict our attention to the range $0 \leqq \vartheta \leqq \pi / 4$.

We deal first with the range $0 \leqq \vartheta \leqq \sin ^{-1}\left(1-r^{2}\right) / r$. Since $\cos \vartheta$ is bounded away from 0 , on this range, the substitution

$$
\sin \vartheta=\frac{1-r^{2}}{2 r} \lambda, \quad \cos \vartheta d \vartheta=\frac{1-r^{2}}{2 r} d \lambda
$$

allows us to replace the integral in question with

$$
K_{1} \int_{0}^{2}(1-r)^{-2} \exp \left\{-\frac{\left(1-r^{2}\right)^{2}\left|1-\lambda^{2}\right|}{K_{2}(1-r)^{4}}\right\} d \lambda
$$

(here $K_{1}$ and $K_{2}$ denote positive constants independent of $r$ ), and if we write $1-r=\mu$, we obtain the upper bounds

$$
\begin{aligned}
& K_{1} \int_{0}^{2} \mu^{-2} \exp \left\{-K_{3}|1-\lambda| \mu^{-2}\right\} d \lambda \\
& \quad=2 K_{1} \int_{0}^{1} \mu^{-2} \exp \left\{-K_{3} \lambda \mu^{-2}\right\} d \lambda<2 K_{1} \int_{0}^{\infty} \exp \left(-K_{3} s\right) d s=2 K_{1} / K_{3} .
\end{aligned}
$$

For the integral from $\vartheta=\sin ^{-1}\left(1-r^{2}\right) / r$ to $\vartheta=\pi / 4$ we have the majorant

$$
K_{4} \int_{\sin ^{-1}\left(1-r^{2}\right) / r}^{\pi / 4}\left(\sin ^{2} \vartheta / 2\right)^{-\frac{2}{2}} \exp \left\{-\frac{3 r^{2} \sin ^{2} \vartheta}{K_{5} \sin ^{4} \vartheta / 2}\right\} d \theta,
$$

and the substitution $\sin \vartheta=t$ shows that this is less than

$$
K_{6} \int_{0}^{\infty} t^{-3} \exp \left(-K_{7} t^{-2}\right) d t=K_{6} / 2 K_{7} .
$$

This concludes the proof that $w$ is a Tsuji function.

To see that the two segments $S(0, \pm \pi / 4$ ) (which make angles $\pm \pi / 4$ with the real axis at $z=1$ ) are segments of Julia, we consider (for example) two segments $S(0, \pi / 4 \pm \varepsilon)$. The function $f$ carries the Stolz angle between these segments into a certain infinite triangle in the right half-plane. The triangle is bounded by portions of two lines through the point $z=-1$ and by the segment of the imaginary axis that lies between them.

The function $g$ carries the same Stolz angle into a domain containing a wedge that in turn contains the imaginary axis, and it follows immediately that the segment $S(0, \pi / 4)$ is a segment of Julia for $w$. This concludes the proof of Theorem 5.

Conjecture 1 . If $w$ is a holomorphic Tsuji function, then at most finitely many points $e^{i s}$ are endpoints of segments of Julia in $D$, for $w$. 
Conjecture 2. If $w$ is a holomorphic Tsuji function, then at most finitely many segments in $D$ are segments of Julia for $w$.

Let $A$ denote one of the two circular arcs in $D$ that meet the circle $C$ at an angle $\pi / 4$ at the two points $z= \pm 1$. Then the function $w$ that we used in the proof of Theorem 5 has the property that $|w(z)|=1$ on $A$, and it followsfurther that $\left|w^{*}(z)\right|=2|1+z| /|1-z|^{3}$ on $A$. By a theorem of O. LeHTo and K.I. VIRTANEN [1, Section 12], we conclude that $w$ is not a normal function in $D$.

Conjecture 3. If $w$ is a holomorphic, normal Tsuji function, then $w$ has no segments of Julia.

\section{References}

[1] LeHTo, O., and K. I. VIRTANEN: Boundary behaviour and normal meromorphic functions. Acta Math. 97, 47-65 (1957).

[2] NoshIRo, K.: Cluster Sets. Ergebnisse der Mathematik und ihrer Grenzgebiete. Berlin-Göttingen-Heidelberg: Springer 1960.

[3] TsuJ, M.: A theorem on the boundary behaviour of meromorphic function in $|z|<1$. Comment. Math. Univ. St. Paul 8, 53-55 (1960). Math. Rev. 22, 1899, No. 11131 (1961).

Lilburn Tower, Alnwick, Northumberland, G.B.

Dept. of Math., University of Michigan, Ann Arbor, Mich., USA.

(Received January 14, 1964) 